\title{
ON SPLITTING FIELDS FOR CERTAIN LIE ALGEBRAS OF PRIME CHARACTERISTIC
}

\author{
RICHARD T. BARNES ${ }^{1}$
}

1. Let $F$ be a field of prime characteristic different from 2 or 3 and $L$ a Lie algebra over $F$ with an abelian Cartan subalgebra $H$. For $\alpha$ in $H^{*}$ (the dual space of $H$ ) set $L_{\alpha}=\{x \in L \mid[x h]=\alpha(h) x$, for all $h$ in $H\}$, and as usual, if $L_{\alpha} \neq(0), \alpha$ is called a root with respect to $H$ and $L_{\alpha}$ the root space for $\alpha$. We have $L_{0}=H$ and $\left[L_{\alpha} L_{\beta}\right] \subset L_{\alpha+\beta}$. Seligman and Mills in [1] have called $L$ a Lie algebra of classical type if $L$ contains an abelian Cartan subalgebra $H$ and if $H$ and $L$ satisfy:

(i) $[L L]=L$.

(ii) $L$ has center (0).

(iii) $L$ is a direct sum of subspaces $L_{\alpha}$.

(iv) If $\alpha$ is a nonzero root, then $\left[L_{\alpha} L_{-\alpha}\right]$ is one-dimensional.

(v) If $\alpha$ is a nonzero root and $\beta \in H^{*}$, then there is a positive integer $m$ such that $\beta+m \alpha$ is not a root.

Let $L$ be a Lie algebra over $F$ such that $L_{K}$ is of classical type, where $K$ is the algebraic closure of $F$. An extension field $P$ of $F$ is called a splitting field for $L$ provided $L_{P}$ is of classical type. We can now state the main theorem of this paper as:

ThEOREM 1.1. Every semisimple Lie algebra over $F$ with nondegenerate Killing form $(x, y)=\operatorname{tr}(\operatorname{ad} x)(\operatorname{ad} y)$ has a separable splitting field.

Note that if $F$ is finite and $L$ has nondegenerate Killing form then every finite extension is separable, in particular one that splits $L$. Therefore, we may assume, in what follows, that $F$ is infinite.

2. LeMma 2.1. If $L$ is semisimple with nondegenerate Killing form, then $L$ contains a regular element $x$ such that the minimum polynomial of $\operatorname{ad}(x)$ has the form:

$$
\mu(\lambda)=\lambda \prod_{\alpha}(\lambda-\alpha(x))
$$

where the $\alpha(x)$ are distinct and different from zero in some extension $P$ of $F$.

Proof. Recall that an element $x$ in $L$ is regular provided the 0 space of $\operatorname{ad}(x)$ has minimal dimension. If $x$ is regular in $L$ and $P$ is

Received by the editors May 7, 1965.

1 This work is part of the author's doctoral dissertation prepared under the direction of Professor George Seligman at Yale University. 
an extension of $F$ then $x$ is regular in $L_{P}$. To see this, let $\left(u_{1}, u_{2}, \cdots, u_{n}\right)$ be a basis for $L$ and $\left(X_{1}, X_{2}, \cdots, X_{n}\right)$ be algebraically independent indeterminants. Let $F^{\prime}=F\left(X_{1}, \cdots, X_{n}\right)$. Then $X=\sum X_{i} u_{i}$ is in $L_{F^{\prime}}$ and the characteristic polynomial of $\operatorname{ad}(X)$ is given by:

$$
\operatorname{det}(\lambda I-\operatorname{ad}(X))=\sum_{0}^{n} M_{i}(X) \lambda^{i},
$$

where $M_{i}(X) \in F\left[X_{1}, \cdots, X_{n}\right]$. If $x=\sum_{1}^{n} \xi_{j} u_{j}$, then $M_{i}(\xi)=0$ for $i<r$, where $r$ is the dimension of the zero space of $\operatorname{ad}(x)$, and $M_{r}(\xi)$ $\neq 0$. Also, since $x$ is regular, $M_{i}\left(\eta_{1}, \eta_{2}, \cdots, \eta_{n}\right)=0$ for $i<r$ and all $\eta_{i}$ in $F$. Thus, since $F$ is infinite, $M_{i}(X)$ is zero for $0 \leqq i<r$ and

$$
\operatorname{det}(\lambda I-\operatorname{ad}(X))=\sum_{r}^{n} M_{i}(X) \lambda^{i} .
$$

Now let $y=\sum \mu_{j} u_{j}, \mu_{j}$ in $P$, i.e. $y$ in $L_{P}$. Then $M_{i}(\mu)=0$ for $0 \leqq i<r$, so that the zero space of $\operatorname{ad}(y)$ in $L_{P}$ has dimension greater than or equal to $r$. Therefore $x$ is regular in $L_{P}$ as claimed. As in the above, for generic element $X$ in $L_{F}$, we have:

$$
\operatorname{det}(\lambda I-\operatorname{ad}(X))=\lambda^{r}\left(M_{r}(X)+M_{r+1}(X) \lambda+\cdots+M_{n}(X) \lambda^{n-r}\right),
$$

where $M_{r}(X) \neq 0$. Consider $g(X, \lambda)$, where:

$$
g(X, \lambda)=M_{r}(X)+M_{r}(X) \lambda+\cdots+M_{n}(X) \lambda^{n-r} .
$$

Then $g(X, \lambda) \in F\left[X_{1}, \cdots, X_{n}, \lambda\right] \subset F\left(X_{1}, \cdots, X_{n}\right)[\lambda]=F^{\prime}[\lambda]$, and thus $g$ has a discriminant given by:

$$
D\left(X_{1}, \cdots, X_{n}\right)=\left(\prod_{i<j}\left(\rho_{i}-\rho_{j}\right)\right)^{2},
$$

where $\rho_{1}, \rho_{2}, \cdots, \rho_{n-r}$ are all roots of $g(X, \lambda)$ as a polynomial in $\lambda$ in some splitting field over $F^{\prime}$, multiple ones taken as many times as their multiplicity. Now, $D\left(X_{1}, \cdots, X_{n}\right)$ is a symmetric function of the roots and therefore is in the ring generated by the elementary functions of the roots, i.e. the ring generated by $M_{r}(X), M_{r+1}(X)$, $\cdots, M_{r-r}(X)$. Thus, there exists a polynomial $Q\left(y_{r}, y_{r+1}, \cdots, y_{n}\right)$ with integral coefficients such that

$$
Q\left(M_{r}(X), \cdots, M_{n}(X)\right)=0
$$

if and only if $g(X, \lambda)$ has repeated roots in its splitting field. Consider $q(X)=M_{r}(X) Q\left(M_{r}(X), \cdots, M_{n}(X)\right)$. Then $q(X) \in F\left[X_{1}, \cdots, X_{n}\right]$ and if $q(X) \neq 0$ there exist elements $\xi_{1}, \cdots, \xi_{n}$ in $F$ such that $q\left(\xi_{1}, \cdots, \xi_{n}\right) \neq 0$. Suppose such an $n$-tuple exists and set $x=\sum \xi_{i} u_{i}$, 
$x \in L$. Then $\operatorname{ad}(x)$ has characteristic polynomial

$$
\operatorname{det}(\lambda I-\operatorname{ad}(x))=\lambda^{r}\left(M_{r}(\xi)+M_{r+1}(\xi) \lambda+\cdots+\lambda^{n-r}\right) .
$$

$M_{r}(\xi) \neq 0$ and $M_{r}(\xi)+M_{r+1}(\xi) \lambda+\cdots+M_{n}(\xi) \lambda^{n-r}$ has distinct roots in a splitting field. Thus $x$ is regular and the minimum polynomial of $\operatorname{ad}(x)$ has the form:

$$
\mu(\lambda)=\lambda \prod_{\alpha}(\lambda-\alpha(x)),
$$

where $\alpha(x)$ are distinct and different from zero. It remains to show that $q(X) \neq 0$. For this, let $H$ be a standard Cartan subalgebra in $L_{K}$ and $h_{0} \in H$ be such that $\alpha\left(h_{0}\right)$ are distinct and nonzero for all roots $\alpha$ relative to $H$. If $h_{0}=\sum \omega_{i} u_{i}, \omega_{i} \in K$, then $q\left(\omega_{1}, \cdots, \omega_{n}\right)$ is not zero, so that $q\left(X_{1}, \cdots, X_{n}\right) \neq 0$, as desired.

3. Proof of Theorem 1.1. Let $L$ be a semisimple Lie algebra over $F$ of dimension $n$, with nondegenerate Killing form, and $x$ a regular element, where the dimension of the zero-space of $\operatorname{ad}(x)$ is $r$ and where the minimum polynomial of $\operatorname{ad}(x)$ has the form:

$$
\mu_{x}(\lambda)=\lambda \prod_{\alpha}(\lambda-\alpha(x)), \quad \alpha(x) \in F,
$$

with all $\alpha(x)$ distinct, different from zero, and $n-r$ in number. We will show that $L$ is of classical type. Note that (ii) is satisfied by our hypotheses. Let $H$ be the zero space of $\operatorname{ad}(x)$. Then $H$ is the Cartan subalgebra of $L$ which will play the role of satisfying the remaining axioms, and $H$ has dimension $r$. Since all $\alpha(x)$ are distinct and characteristic roots of $\operatorname{ad}(x)$, the subspaces $L_{\alpha(x)}$ corresponding to $\alpha(x)$ have dimension one. Then we have

$$
L=H+\sum_{\alpha} L_{\alpha}
$$

Now for $h \in H,[h x]=0$ and if $y \in L_{\alpha},[[y h] x]=[[y x] h]=\alpha(x)[y h]$. Thus $[y h] \in L_{\alpha}$, i.e. $\left[L_{\alpha} H\right] \subseteq L_{\alpha}$ for $\alpha(x) \neq 0$. Since $L_{\alpha}$ is one dimensional this means that for $e_{\alpha} \in L_{\alpha}$ and $h \in H,\left[e_{\alpha} h\right]=\lambda(h) e_{\alpha}$. Set $\alpha(h)$ $=\lambda(h)$. Thus the characteristic roots of $\operatorname{ad}(h)$ are in the ground field and $L_{\alpha}$ is a root space relative to $H$. Furthermore, the restriction to $H$ of the Killing form on $L$ is nondegenerate. To see this, let $h \in H$, $e_{\alpha} \in L_{\alpha}$, for $\alpha \neq 0$. Then $\left[e_{\alpha} h\right]=\alpha(h) e_{\alpha}$ and we can choose $e_{\alpha}^{(1)} \in L_{\alpha}$ such that $\left[e_{\alpha}^{(1)} h\right]=e_{\alpha}$. Then $\left(h, e_{\alpha}\right)=\left(h,\left[e_{\alpha}^{(1)} h\right]\right)=\left([h h], e_{\alpha}^{(1)}\right)=0$. Thus $\left(H, L_{\alpha}\right)=0$ for all $\alpha \neq 0$ and the form must be nondegenerate on $H$. It follows that $H$ is abelian. In the case where $F$ is algebraically closed 
this is a result due to Zassenhaus. In the general case a field extension argument gives the result. This, together with (2) now shows that axiom (iii) holds.

Thus we have that $L$ contains an abelian Cartan subalgebra $H$, and that relative to a fixed basis for $L, \operatorname{ad}(h)$ has a diagonal matrix for every $h \in H$. Next we note that if $\alpha$ is a root different from zero, then so is $-\alpha$. For, let $e_{\alpha} \in L_{\alpha}, e_{\beta} \in L_{\beta}$. Then, for some $h \in H, e_{\alpha}=\left[e_{\alpha} h\right]$, and $\left(e_{\beta}, e_{\alpha}\right)=\left(e_{\beta},\left[e_{\alpha} h\right]\right)=\left(\left[e_{\beta} e_{\alpha}\right], h\right)=0$, unless $\beta=-\alpha$, since $\left[e_{\beta} e_{\alpha}\right]$ $\in L_{\alpha+\beta}$. If $L_{-\alpha}=(0)$ we have $\left(L, e_{\alpha}\right)=0$, a contradiction.

The nondegeneracy of the form $(x, y)$ on $H$ implies that for each $\alpha \in H^{*}$ there exists an $h_{\alpha} \in H$ such that $\left(h_{\alpha}, h\right)=\alpha(h)$, for all $h \in H$.

In what follows we shall have occasion to refer to results in Seligman's Memoir [2] which we shall denote by $M$.

Lemma 3.1 (M, Corollary 3.2). If $e_{\alpha} \in L_{\alpha}, e_{-\alpha} \in L_{-\alpha}$, then $\left[e_{-\alpha} e_{\alpha}\right]$ $=\left(e_{-\alpha}, e_{\alpha}\right) h$.

Since $L$ has nondegenerate form, every derivation of $L$ is inner [3]. Thus, for $x \in L, \operatorname{ad}(x)^{p}$ is a derivation in $L$ and there exists a unique $y \in L$ such that $\operatorname{ad}(x)^{p}=\operatorname{ad}(y)$. Setting $x^{p}=y, L$ becomes a restricted Lie algebra over $F$ and the adjoint mapping is a restricted representation of $L$.

We turn now to a modification of two results due to Jacobson dealing with low-dimensional Lie algebras and their representations $(M$, Lemma 4.1 and 4.2). The modification involves replacing algebraic closure of the ground field with the fact that for the representation $U$ we have $U(h)$ is diagonalizable for all $h \in H$.

Lemma 3.2. Let L be a two-dimensional Lie algebra over $F$ with basis elements $e, h$, and $[e h]=e$. Let $U$ be an irreducible representation of $L$ such that $U(h)$ and $U(e)^{p}$ are diagonalizable. Then either $U(e)^{p}=0$ or $U$ is equivalent to the $p$-dimensional representation $W$ :

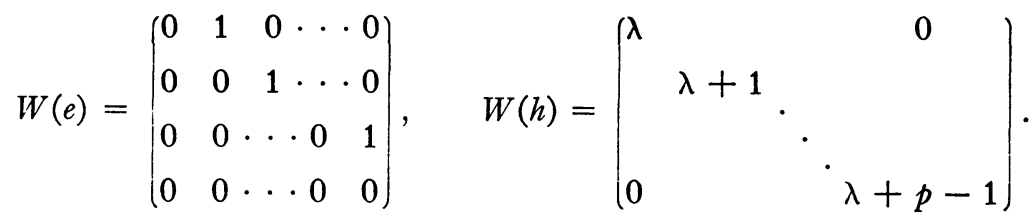

Proof. Suppose $U(e)^{p}=U\left(e^{p}\right) \neq 0$. Then, since $U(e)^{p}$ and $U(h)^{p}$ $-U(h)$ are diagonalizable by our assumptions, these matrices are scalar. For, if $V_{1}=\left\{v \mid v U(e)^{p}=\lambda v\right\}$, and $V_{2}=\left\{v \mid v\left(U(h)^{p}-U(h)\right)=\mu v\right\}$, then these are invariant subspaces of the representation space $V$ and since one of them is not zero for some $\lambda$ by diagonalizability one must be the whole space. Thus in each case, $U(e)^{p}=\sigma I, \sigma \in F$, 
and $U(h)^{p}-U(h)=\rho I, \rho \in F$. Now, let $\lambda$ be a characteristic root of $U(h)$ and $v \neq 0$ such that $v U(h)=\lambda v$. Then $v U(e)^{p}=\sigma v$, and the space spanned by $\left\{v, v U(e), \cdots, v U(e)^{p-1}\right\}$ is an invariant subspace of dimension $p$, thus the whole space $V$. To see the invariance, we note:

$$
v U(e)^{k} U(h)=(\lambda+k) v U(e)^{k}, \quad 0 \leqq k \leqq p-1 .
$$

Now, relative to this basis for $V$, the matrices of $U(h)$ and $U(e)$ have the form of the lemma.

Lemma 3.3. Let $L$ be a three-dimensional Lie algebra over $F$ with basis $e, f, h$ and let $[e f]=h,[f h]=0=[e h]$. Let $U$ be a nonzero irreducible representation of $L$ such that $U(e)^{p}=0$ and $U(f)^{p}=0$ and $U(h)$ is diagonalizable. Then $\operatorname{tr}(u(e) U(f))=0$.

Proof. Since $U(h)$ is diagonalizable and centralizes the representation we have $U(h)$ is scalar, i.e. $U(h)=\lambda I$. If $\lambda=0$, then $U(e) U(f)$ $=U(f) U(e)$. Since both $U(e)$ and $U(f)$ are nilpotent, so is $U(e) U(f)$, and thus $\operatorname{tr}(U(e) U(f))=0$. Suppose now that $\lambda \neq 0$ and let $v \neq 0$ be an element of the representation space $V$ such that $v U(f)=0$. Such a $v$ exists since $U(f)$ is nilpotent. Now, let $K$ be the space spanned by $\left\{v, v U(e), \cdots, v U(e)^{p-1}\right\}$. Then $K U(e) \subset K$ and $K U(h) \subset K$. Furthermore, we have:

$$
v U(e)^{k} U(f)=v U(e)^{k-1} U(f) U(e)+v U(e)^{k-1} U(h) .
$$

Actually, by induction we have:

$$
v U(e)^{k} U(f)=k \lambda\left(v U(e)^{k-1}\right), \quad k \geqq 1 .
$$

Thus, $K=V$, the whole space, and thus $\left\{v, v U(e), \cdots, v U(e)^{p-1}\right\}$ is a basis for $V$. The matrices relative to this basis are:

$$
U(e)=\left(\begin{array}{lllll}
0 & 1 & 0 & \cdots & 0 \\
0 & 0 & 1 & \cdots & 0 \\
0 & 0 & 0 & \cdots & 1 \\
0 & 0 & 0 & \cdots & 0
\end{array}\right), \quad U(f)=\left(\begin{array}{ccccc}
0 & 0 & 0 & \cdots & 0 \\
\lambda & 0 & 0 & \cdots & 0 \\
0 & 2 \lambda & \cdots & 0 \\
0 & 0 & \cdots(p-1) \lambda & 0
\end{array}\right)
$$

and thus $\operatorname{tr}(U(e) U(f))=0$ as claimed.

Using these lemmas together with our diagonalizability condition we can now prove the following analogues of the required theorems in $\mathrm{M}$.

Theorem 3.1 (M, TheOREM 4.1). If $\alpha \neq 0$ is a root then $e_{\alpha}^{p}=0$.

Proof. For $h \in H,\left[h e_{\alpha}^{p}\right]=0$, thus $e_{\alpha}^{p} \in H$. Choose $h$ such that $\alpha(h)$ $=1$. Then $\left\{e_{\alpha}, h\right\}$ forms a two-dimensional Lie algebra $L_{1}$ as in Lemma 3.2. For the representation $U(x)$ take $\operatorname{ad}_{L}(x)$. Then, the restriction of $U$ to $L_{1}$ can be written in the form: 


$$
\left(\begin{array}{ccccc}
U_{1} & & & & \\
& U_{2} & & 0 \\
* & & \cdot & \\
& & & \cdot \\
& & & U_{t}
\end{array}\right) .
$$

Furthermore, since $\operatorname{ad}_{L}(h)$ are diagonal for $h \in H$, the same holds for $\operatorname{ad}_{L}(h)$ restricted to $M, M$ an irreducible $L_{1}$ submodule of $L$, and for the transformations induced by $\operatorname{ad}_{L}(h)$ in $L / M$. Continuing this argument on $L / M$ we see that $U_{i}(h)$ is a diagonal matrix relative to a suitable basis for each $h \in H$.

Now $\left(e_{\alpha}^{p}, h\right)=\operatorname{tr}\left(U\left(e_{\alpha}\right)^{p} U(h)\right)$ and either $U_{i}\left(e_{\alpha}\right)^{p}=0$ or $U_{i}\left(e_{\alpha}\right)$ has the form of Lemma 3.2. In any case, $\operatorname{tr}\left(U_{i}\left(e_{\alpha}\right)^{p} U_{i}(h)\right)$ is zero so that $\left(e_{\alpha}^{p}, h\right)=0$. This holds whenever $\alpha(h) \neq 0$. If $\alpha(h)=0$, let $h_{1} \in H$ be chosen such that $\alpha\left(h_{1}\right) \neq 0$. Then $\alpha\left(h+h_{1}\right) \neq 0$ and $\left(e_{\alpha}^{p}, h\right)=\left(e_{\alpha}^{p}, h+h_{1}\right)$ $-\left(e_{\alpha}^{p}, h_{1}\right)=0$. Thus, $\left(e_{\alpha}^{p}, H\right)=0$, which gives $e_{\alpha}^{p}=0$.

Theorem 3.2 (M, Theorem 4.2). If $\alpha \neq 0$ is a root, then $\alpha\left(h_{\alpha}\right) \neq 0$.

Proof. Suppose $\alpha\left(h_{\alpha}\right)=0$ and $e_{\alpha} \neq 0, e_{\alpha} \in L_{\alpha}, e_{-\alpha} \neq 0, e_{-\alpha} \in L_{-\alpha}$ such that $\left(e_{\alpha}, e_{-\alpha}\right)=1$. By Lemma $3.1\left[e_{-\alpha} e_{\alpha}\right]=h_{\alpha}$. Let $L_{1}$ be the algebra spanned by $\left\{e_{\alpha}, e_{-\alpha}, h_{\alpha}\right\}$. For the irreducible constituent $U_{i}$ of the restriction of $U=\operatorname{ad}_{L}$ to $L_{1}$ we have $U_{i}\left(e_{\alpha}\right)^{p}=0$ and $U_{i}\left(e_{-\alpha}\right)^{p}=0$. Thus by Lemma 3.3 we obtain $\operatorname{tr}\left(U_{i}\left(e_{-\alpha}\right) U_{i}\left(e_{\alpha}\right)\right)=0$. Therefore, $\operatorname{tr}\left(U\left(e_{-\alpha}\right) U\left(e_{\alpha}\right)\right)=0$, i.e. $\left(e_{-\alpha}, e_{\alpha}\right)=0$, a contradiction.

Now, by Lemma 3.1 and Theorem 3.2 we have $\left[L_{\alpha} L_{-\alpha}\right]$ is one dimensional, giving us axiom (iv). Theorems 3.1 and 3.2 make it possible now to use the results of $\S 5$ of $\mathrm{M}$. (For a complete proof of $\mathrm{M}$, Lemma 5.1 see [1].) In particular, axiom (v) for our algebras is a consequence of Theorems 5.2 and 5.4 in $\mathrm{M}$.

Finally, for axiom (i), we note that $[L L]_{K}=\left[L_{K} L_{K}\right]=L_{K}, K$ the algebraic closure of $F$. Hence we have:

$$
\operatorname{dim}_{F}[L L]=\operatorname{dim}_{K}[L L]_{K}=\operatorname{dim}_{K} L_{K}=\operatorname{dim}_{F} L .
$$

Therefore, $[L L]=L$. Thus, our algebra $L$ is of classical type and this together with Lemma 2.1 proves Theorem 1.1.

\section{BIBLIOGRAPHY}

1. W. H. Mills and G. B. Seligman, Lie algebras of classical type, J. Math. Mech. 6 (1957), 519-548.

2. G. B. Seligman, On Lie algebras of prime characteristic, Mem. Amer. Math. Soc. No. 19 (1956), 85 pp.

3. H. Zassenhaus, Über Lie'sche ringe mit primzahlcharakteristik, Abh. Math. Sem. Hamburg 13 (1939), 1-100.

The Ohio State University 